\title{
Robot-assisted procedures in pediatric neurosurgery
}

\author{
Alessandro De Benedictis, MD, PhD, ${ }^{1}$ Andrea Trezza, MD, ${ }^{1,2}$ Andrea Carai, MD, PhD, ${ }^{1}$ \\ Elisabetta Genovese, DMP, ${ }^{3}$ Emidio Procaccini, MD, ${ }^{1}$ Raffaella Messina, MD, ${ }^{1}$ \\ Franco Randi, MD, PhD, ${ }^{1}$ Silvia Cossu, MD,' Giacomo Esposito, MD,1 Paolo Palma, MD,1 \\ Paolina Amante, MD, ${ }^{1}$ Michele Rizzi, MD, ${ }^{4,5}$ and Carlo Efisio Marras, MD ${ }^{1}$
}

'Department of Neuroscience and Neurosurgical Unit and ${ }^{3}$ Enterprise Risk Management, Medical Physics Department, Bambino Gesù Children's Hospital, IRCCS, Rome; ${ }^{2}$ Neurosurgery, Department of Surgery and Translational Medicine, Milan Center for Neuroscience, University of Milano-Bicocca, San Gerardo Hospital, Monza; 4"Claudio Munari” Center for Epilepsy Surgery, Niguarda Hospital, Milan; and ${ }^{5}$ Department of Neuroscience, University of Parma, Italy

\begin{abstract}
OBJECTIVE During the last 3 decades, robotic technology has rapidly spread across several surgical fields due to the continuous evolution of its versatility, stability, dexterity, and haptic properties. Neurosurgery pioneered the development of robotics, with the aim of improving the quality of several procedures requiring a high degree of accuracy and safety. Moreover, robot-guided approaches are of special interest in pediatric patients, who often have altered anatomy and challenging relationships between the diseased and eloquent structures. Nevertheless, the use of robots has been rarely reported in children. In this work, the authors describe their experience using the ROSA device (Robotized Stereotactic Assistant) in the neurosurgical management of a pediatric population.
\end{abstract}

METHODS Between 2011 and 2016, 116 children underwent ROSA-assisted procedures for a variety of diseases (epilepsy, brain tumors, intra- or extraventricular and tumor cysts, obstructive hydrocephalus, and movement and behavioral disorders). Each patient received accurate preoperative planning of optimal trajectories, intraoperative frameless registration, surgical treatment using specific instruments held by the robotic arm, and postoperative CT or MR imaging.

RESULTS The authors performed 128 consecutive surgeries, including implantation of 386 electrodes for stereo-electroencephalography (36 procedures), neuroendoscopy (42 procedures), stereotactic biopsy ( 26 procedures), pallidotomy (12 procedures), shunt placement (6 procedures), deep brain stimulation procedures (3 procedures), and stereotactic cyst aspiration ( 3 procedures). For each procedure, the authors analyzed and discussed accuracy, timing, and complications.

CONCLUSIONS To the best their knowledge, the authors present the largest reported series of pediatric neurosurgical cases assisted by robotic support. The ROSA system provided improved safety and feasibility of minimally invasive approaches, thus optimizing the surgical result, while minimizing postoperative morbidity.

https://thejns.org/doi/abs/10.3171/2017.2.FOCUS16579

KEY WORDS pediatric neurosurgery; robotics; minimally invasive neurosurgery

$\mathrm{S}$ INCE the early 1980s, robotic technology has evolved, starting from its initial use in the industrial sector to its use within medical and surgical practice. Over the next 3 decades, the use of medical robots has rapidly expanded to several specific fields, such as soft-tissue, urological, orthopedic, general laparoscopic, and cardiac surgeries. $^{38,45,56}$

Robotic assistance was developed in neurosurgical procedures with the aim of improving feasibility and effec- tiveness of several procedures that require a high level of accuracy and safety, such as biopsy, ${ }^{36}$ neuroendoscopy, ${ }^{8,69,71}$ radiosurgery, ${ }^{2}$ neuromodulation treatments, ${ }^{43,45}$ and other stereotactic procedures. ${ }^{7,37}$ Recently, more sophisticated systems have been proposed for other complex treatments, such as brain tumor removal,4,35,40,42,61,62 deep electrode placement for stereo-electroencephalography (SEEG) recording, ${ }^{14,15,16,29}$ laser ablation in medically intractable epilepsy, ${ }^{13,30,50}$ and other functional approaches. ${ }^{66}$

ABBREVIATIONS DBS = deep brain stimulation; EPLE = entry point localization error; GPI = globus pallidus internus; $\mathrm{HH}=$ hypothalamic hamartoma; ROSA = Robotized Stereotactic Assistant; SEEG = stereo-electroencephalography; TPLE = target point localization error.

SUBMITTED December 31, 2016. ACCEPTED February 21, 2017.

INCLUDE WHEN CITING DOI: 10.3171/2017.2.FOCUS16579. 
In this context, robotic technology is continuously improving, mainly in terms of versatility, adaptability, geometric accuracy, stability, repeatability, integration of extensive information, safety, dexterity, tactile feedback, and hand-eye coordination. The main goal of this technology is to enhance the surgeon's skills so that he or she can perform microsurgical manipulations, minimally invasive access procedures, accurate stereotactic approaches, and image-guided procedures. In particular, 3 possible surgeon-robot interaction modalities have been described, depending on whether the robot performs specific preplanned motions under the supervision of the surgeon (supervisory-controlled system), if the surgeon directly controls the robotic manipulator through a haptic interface (telesurgical system), or if the robot and surgeon jointly control the surgical instrument (shared-control system). ${ }^{48}$

Moreover, robot-guided methods are of special interest in pediatric neurosurgery. In fact, many brain diseases that occur during childhood, such as hydrocephalus, epilepsy, tumors, and movement and behavioral disorders, often constitute a particular challenge for neurosurgeons. Developing structures are more vulnerable to injury than the developed structures in adults, especially with regard to small and deep targets. ${ }^{65}$ In addition, the normal anatomy of a child's brain is often altered by specific constitutional factors (e.g., malformations) or by the disease itself. Consequently, neurosurgical management of these cases requires careful preoperative planning and a high degree of intraoperative precision to correctly identify and reach the surgical target without damaging the surrounding eloquent neurovascular structures.

Nevertheless, robot-assisted approaches have been reported infrequently in pediatric patients. Moreover, the cases described in the literature mainly concern urological, abdominal, and cardiac experiences, with rare anecdotal reports on neurosurgical applications..$^{1,69}$

Among the robotic systems currently used, the Robotized Stereotactic Assistant (ROSA, Medtech) is a recently developed image-guided device that provides guidance for spatial positioning and orientation of several neurosurgical instruments according to a planned trajectory.

In this work, we report on 116 pediatric patients who underwent surgical procedures for a variety of diseases with the assistance of the ROSA system at the Neurosurgery Unit of the Bambino Gesù Children's Hospital, in Rome, Italy.

\section{Methods}

\section{Patient Population}

We retrospectively evaluated 116 consecutive pediatric patients (63 males and 53 females; mean age at surgery 11.2 years), who, between 2011 and 2016, underwent a total of 128 robot-assisted procedures for the treatment of various diseases at our institution. The diagnoses are summarized in Table 1.

\section{Preoperative Planning}

All patients underwent 3D Gd-enhanced T1-weighted MRI. For some specific procedures, such as pallidotomy,
TABLE 1. Summary of diagnoses in 116 patients undergoing robot-assisted surgery

\begin{tabular}{cc}
\hline \multicolumn{1}{c}{ Diagnosis } & No. of Patients \\
\hline Drug-resistant epilepsy & 52 \\
\hline HH & 20 \\
\hline Brain tumor & 40 \\
\hline Basal ganglia/diencephalon tumor & 11 \\
\hline Brainstem tumor & 10 \\
\hline Pineal region tumor & 4 \\
\hline Temporal/temporomesial tumor & 4 \\
\hline Optic pathway glioma & 3 \\
\hline Sellar region tumor & 3 \\
\hline Intraventricular tumor & 3 \\
\hline Corpus callosum tumor & 1 \\
\hline Multilobar tumor & 1 \\
\hline Dystonia & 13 \\
\hline Obstructive hydrocephalus & 6 \\
\hline Arachnoid cyst & 2 \\
\hline Drug-resistant aggressive behavior & 1 \\
\hline Diffuse white matter degenerative disease & 1 \\
\hline Cerebral proliferative angiopathy & 1 \\
\hline Total no. & 116 \\
\hline
\end{tabular}

deep brain stimulation (DBS), and hypothalamic hamartoma $(\mathrm{HH})$ disconnection, we also used 3D T2-weighted and/or inversion recovery sequences to improve visualization of the basal ganglia and midline structures. To optimize skin rendering, a preoperative volumetric CT scan was performed in all patients.

The CT and MR images were imported to the dedicated planning software and coregistered using a rigid and linear algorithm. The registration accuracy was validated by checking the correspondence of anatomical landmarks (ventricles, bone, venous sinus, and commissures) between the selected sequences. The entry and target points and the trajectory were selected on different possible views (axial, coronal, sagittal, perpendicular and along the trajectory, and anterior commissure-posterior commissure coordinates). Once completed, the planning was copied into the same software on the robotic device.

\section{Intraoperative Phase}

The ROSA system is composed of a platform, including a compact robotic arm and a touch screen, mounted on a mobile trolley. A mechanical arm fixes the robot to the 3 -pin support applied on the patient's head. The position of the head depends on the procedure: neutral, slightly flexed in endoventricular procedures, pallidotomy, and DBS; and slightly rotated in intracranial electrode implantation for SEEG.

We used 2 types of registration: fiducial marker registration using invasive bone fiducials for DBS cases, and frameless surface registration for all other procedures. The first is based on the recognition by a special pointer of the tip of 5 screws that were previously implanted in the patient's skull. The surface registration includes different 
TABLE 2. Summary of 128 procedures, performed by using the ROSA robot

\begin{tabular}{|c|c|c|c|c|c|c|}
\hline \multirow[b]{2}{*}{ Procedure } & \multirow[b]{2}{*}{ No. of Cases } & \multirow{2}{*}{$\begin{array}{l}\text { Mean Op } \\
\text { Time (mins) }\end{array}$} & \multicolumn{2}{|c|}{ Accuracy (mm) } & \multirow[b]{2}{*}{ No. of Failures/Complications } & \multirow{2}{*}{$\begin{array}{l}\text { Mean FU } \\
\text { (mos) }\end{array}$} \\
\hline & & & EPLE & TPLE & & \\
\hline $\begin{array}{l}\text { Electrode implan- } \\
\text { tation }\end{array}$ & 36 (386 electrodes) & 204.53 & $\begin{array}{l}\text { Median } 1.5 \text { (mean } \\
1.59 \pm 1.1)\end{array}$ & $\begin{array}{l}\text { Median } 1.96 \text { (mean } \\
2.22 \pm 1.71)\end{array}$ & 0 & 28.8 \\
\hline $\begin{array}{l}\text { Stereotactic } \\
\text { biopsy }\end{array}$ & 26 & 107.3 & & No error & $\begin{array}{l}\text { Not diagnostic }(n=1) \text {; transient monopa- } \\
\quad \text { resis }(n=2)\end{array}$ & 28.8 \\
\hline $\begin{array}{l}\text { Stereotactic cyst } \\
\text { aspiration }\end{array}$ & 3 & 118.3 & & No error & 0 & 9.6 \\
\hline HH disconnection & 24 & 139.5 & & & $\begin{array}{l}\text { Transient deficits }(n=3) \text {; hemiparesis }(n= \\
\text { 1); hormonal imbalance }(n=2)\end{array}$ & 40.8 \\
\hline Endoscopy* & 18 & 150.8 & & & 0 & 34.8 \\
\hline Shunt placement & 6 & 123.5 & & & Shunt not placed (tough cystic wall; $n=1$ ) & 38.4 \\
\hline Pallidotomy & 12 & 287.7 & & $\begin{array}{l}\text { Median } 1.33 \text { (mean } \\
\qquad 1.45 \pm 0.32 \text { ) }\end{array}$ & 0 & 40.8 \\
\hline DBS & 3 (6 electrodes) & 415 & & $\begin{array}{l}\text { Median } 1.60 \text { (mean } \\
\quad 1.56 \pm 0.35)\end{array}$ & Infection $(n=1)$ & 22.8 \\
\hline Total & 128 & 172.7 & & & 3 failures $/ 5$ transient deficits & 30.6 \\
\hline
\end{tabular}

steps, namely: 1) arm calibration; 2) acquisition of facial landmarks by a contactless distance sensor held by the robot arm (midline frontal, bilateral lateral frontoorbital region, internal and external eye canthus, nasion, and tip of the nasal bone); 3) correction of registration errors by matching the intraoperative scanned 3D volume with the preoperative 3D MRI data; 4) automatic scanning of the relevant areas of the patient's face; 5) manual scanning (lateral surface of the nose and lateral frontoorbital region bilaterally); 6) trajectory feasibility verification according to the selected tool (sensor of distance, endoscope holder, microdescender holder); and 7) final verification of the correspondence between the patient and neuroimaging, by manually positioning the robot arm at the level of the initial facial landmarks.

During the operative phase, the robot arm moves to the selected entry point and is oriented according to the corresponding trajectory. The arm movement can be controlled by the surgeon in 3 possible modes (axial, isocentric, and free) and 2 speeds (slow or fast). The robot arm has $6 \mathrm{df}$ and has haptic features, based on force sensing and feedback.

Several instruments can be connected to a specific holder at the end of the arm, depending on the surgical procedure to be performed. During SEEG, the robot supported different specific instruments (a skull drill, monopolar instruments, and a screwdriver). After checking the correct length, each electrode was introduced and fixed to the skull with a 20- or 25-mm screw. For endoscopic approaches, a bur hole was made according to the planned entry point. A rigid $30^{\circ}$ endoscope (medium size, Karl Storz), integrated with the robot arm was moved from the cortex to the ventricles under navigation control. Once the ventricle was reached in iso-axial mode, the endoscope was moved to the target point by switching to the cooperative or free mode. Third ventriculostomy was performed using a 3-Fr Fogarty balloon-tipped catheter. Disconnec- tion of HHs was performed using a 2- $\mu$ m thulium laser device (RevoLix) or by monopolar cauterization. Biopsies were performed using a Nashold needle (Medtronic). For DBS procedures and pallidotomy, the robot arm supported a microdescender that guided the electrode for neurophysiological mapping, microstimulation, and the final DBS electrode at target. Pallidotomy was performed using a specific electrode during repeated thermocoagulation sessions $\left(70^{\circ} \mathrm{C}\right.$ for 60 seconds, Cosman radiofrequency generator). After surgery, all patients underwent control CT or MR imaging to verify the target and exclude surgical complications.

\section{Results}

We performed 128 neurosurgical procedures assisted by the ROSA system. Eleven of 128 surgeries have been repeated in 10 patients, including SEEG implantation (4 cases), pallidotomy (1 case), HH disconnection (4 procedures), stereotactic biopsy (1 case).

The overall time, including registration and surgical phases, was 172.7 minutes. The mean follow-up was 30.6 months (range 9.6-40.8 months). The procedure was ineffective in 3 cases $(2.4 \%)$, namely, 1 tumor biopsy, 1 intracystic catheter placement, and 1 DBS surgery. Five patients $(3.9 \%)$ had a transient postoperative deficit. No permanent deficit was noted. The main results of each procedure are summarized in Table 2.

\section{Intracranial SEEG Electrode Implantation}

We implanted a total of 386 electrodes (mean 10.7 electrodes per patient, range 6-16) in 36 patients suffering from drug-resistant epilepsy (Fig. 1). The mean surgical time was 204.3 minutes. No intracranial bleeding was observed postoperatively.

To assess the accuracy of electrode positioning, we measured the entry point localization error (EPLE) and 


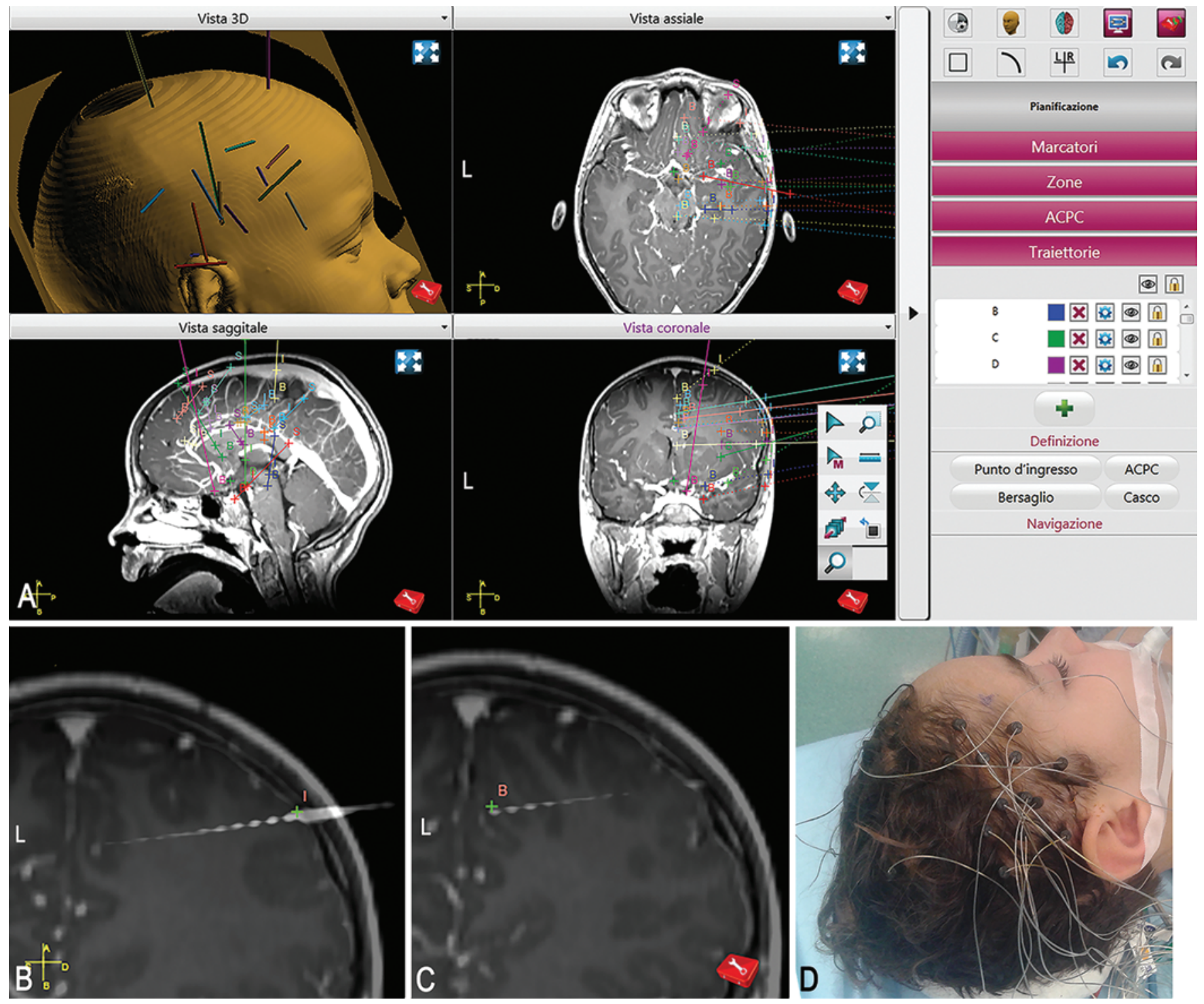

FIG. 1. Intracranial implantation of electrodes for SEEG. A: Screenshot of the preoperative planning. The imported images can be visualized according to different projections. To avoid hemorrhagic complications, the safest trajectories are built and verified on the Gd T1-weighted sequence. The 3D rendering gives a useful general overview of the trajectories; the surgeon can verify the correct distance between the electrodes (left). Different tools for both the image rendering and trajectory planning (right). B and C: Coregistration of the MR image with the postoperative CT verification of the correspondence between the planned trajectory and the implanted electrode, especially at the entry point $(B)$ and the target point $(C)$. D: Intraoperative photograph of the final implantation.

the target point localization error (TPLE). As previously described, EPLE is defined by calculating the Euclidean distance between the planned entry point and the major axis of the implanted electrode on postoperative CT scanning. Similarly, TPLE is defined as the Euclidean distance between the planned target and the major axis of the electrode at its tip. The Euclidean distance between 2 points is defined as the square root of the sum of the squares of the differences between the corresponding coordinates of the points on the 3 axes (x, y, and $\mathrm{z}){ }^{17}$ In our series, we reported a median EPLE of $1.50 \mathrm{~mm}$ (mean $1.59 \pm 1.10 \mathrm{~mm}$ $[ \pm \mathrm{SD}])$, with EPLE greater than $2 \mathrm{~mm}$ in 116 electrodes (30\%). The median TPLE of each electrode was $1.96 \mathrm{~mm}$ (mean $2.22 \pm 1.71 \mathrm{~mm}$ ), with TPLE greater than $2 \mathrm{~mm}$ in
162 electrodes $(42.0 \%)$. When considering only the more recent 10 cases, the median EPLE and TPLE were 1.12 $\mathrm{mm}$ (mean $1.09 \pm 0.8 \mathrm{~mm}$ ) and $1.50 \mathrm{~mm}$ (mean $1.84 \pm 1.91$ $\mathrm{mm}$ ), respectively.

\section{Endoscopic Procedures}

A total of 42 endoscope-assisted procedures were performed to treat different diseases, with a mean surgical time of 144 minutes (Figs. 2 and 3). In particular, 7 patients underwent third ventriculostomy (5 cases) or septostomy (2 cases) for obstructive hydrocephalus. An endoscopic approach was also used to perform 8 endoscope-assisted biopsies of intraventricular or midline tumors, and for fenestration of intraventricular or extraventricular cysts (3 


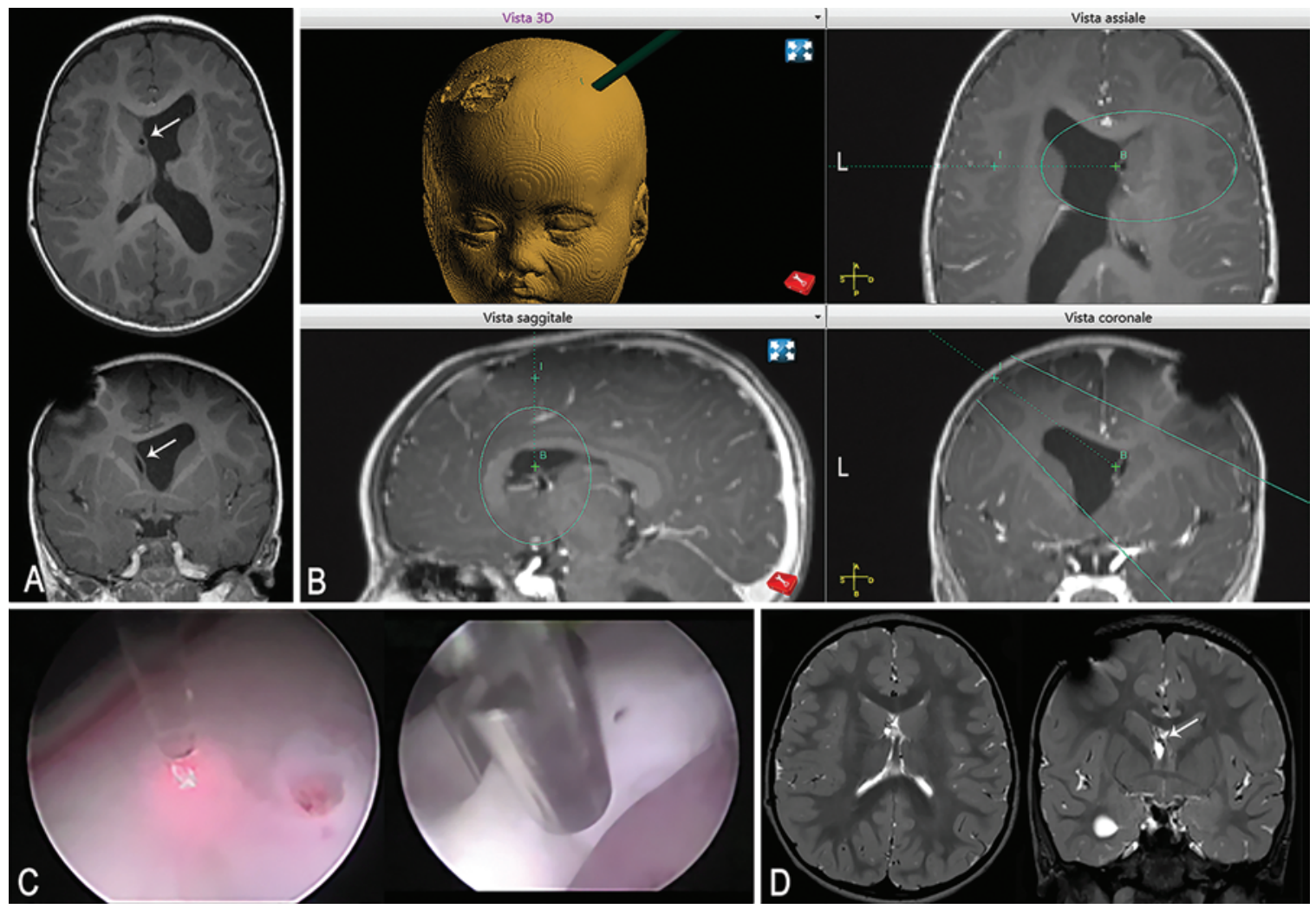

FIG. 2. Endoscopic septostomy in a 4-year-old girl who experienced malfunction of a right-sided ventriculoperitoneal shunt that had been implanted at birth for a postinfection hydrocephalus. A: Preoperative axial (upper) and coronal (lower) MR images showing the ventricular catheter (arrows) and an asymmetry between the lateral ventricles. B: We planned a robot-assisted transventricular septostomy, and we selected the target point at the level of the right-sided catheter. C: Intraoperative endoscopic images showing the laser septostomy (left) and the relocation of the shunt within the left ventricle (right). D: Postoperative axial (left) and coronal (right) MR images demonstrating the resolution of the ventricular asymmetry and the correct position of the shunt (arrow).

cases). The mean size of the lateral ventricle, measured at the frontal horn considering an axial plane from the inner margin of the medial ventricular wall to the inner margin of the lateral wall, was $4.64 \mathrm{~mm}$ for the right ventricle and $5.1 \mathrm{~mm}$ for the left ventricle. No postoperative morbidity was noted in these patients.

Twenty-four endoscopic procedures were performed to disconnect the $\mathrm{HH}$ in 20 patients suffering from drugresistant epilepsy. Because of the epilepsy recurrence, the procedure was repeated twice in 2 patients, and 3 times in 1 patient. The mean surgical time was 139.5 minutes. The early surgical complication rate was $12.5 \%$, due to left hemiparesis (1 case), inappropriate antidiuretic hormone secretion (1 case), and postoperative hypernatremia (1 case). All these patients had complete clinical resolution after 1 month from surgery.

\section{Stereotactic Biopsies}

A total number of 26 robot-assisted stereotactic biopsies were performed in different brain regions, including the basal ganglia/diencephalon region (8 cases), the brainstem (10 cases), the pineal region (2 cases), and other supratentorial lobar territories (6 cases) (Fig. 4). The mean trajectory length was $92 \mathrm{~mm}$. The mean procedural time was 110.6 minutes. We did not find any significant TPLE on postoperative imaging, and diagnosis based on histo- logical results was obtained in 25 of 26 cases (tumor in 24 cases and diffuse white matter degenerative disease in 1 case). In the remaining case, the biopsy sample was insufficient for histopathological diagnosis. Early transient postoperative monoparesis developed in 2 (8\%) biopsy cases (tumor within the globus pallidus in one patient and diffuse intrinsic pontine glioma in another patient). No long-term clinical consequences were noted.

\section{Other Stereotactic Procedures}

We used the ROSA device to place a catheter connected to a subcutaneous reservoir for the aspiration of tumor cysts (5 cases) and CSF shunting (1 case of intracranial hypertension due to diffuse proliferative angiopathy), with a mean time of 124 minutes. In 1 case, the intracystic catheter placement failed because of a tough cystic wall. Three patients underwent stereotactic cyst aspiration through the biopsy needle, with a mean time of 118 minutes. No surgical complications were noted in this series.

\section{Functional Procedures}

We performed 2 bilateral globus pallidus internus (GPI) DBS procedures in 2 cases of primary dystonia and 1 bilateral DBS procedure in the posterior hypothalamus in 1 patient with drug-resistant aggressive behavior and autism 


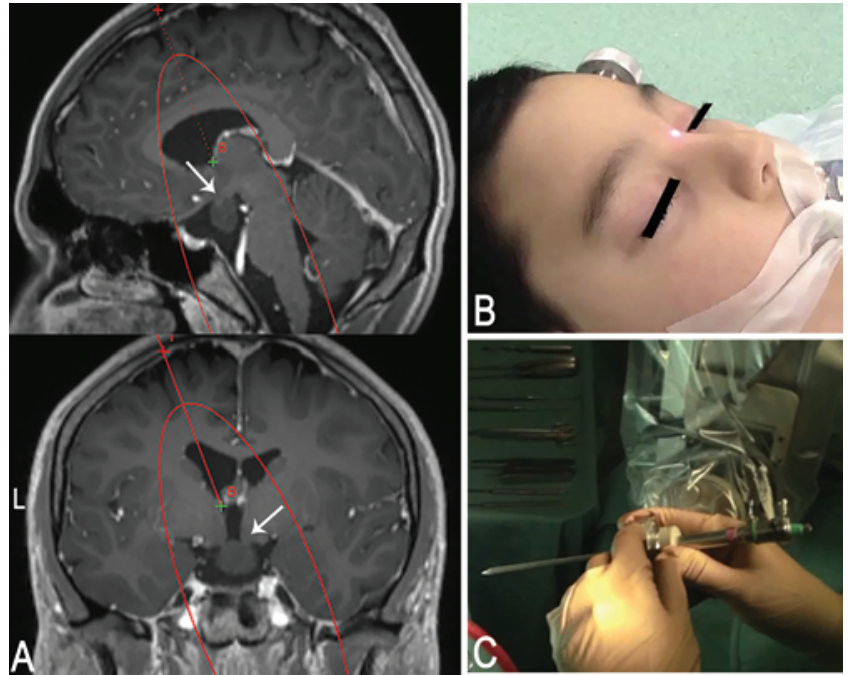

FIG. 3. Transventricular endoscopic disconnection of an $\mathrm{HH}$ in a 6-yearold boy. A: Given the bilateral HH implantation (arrows), we selected a trajectory throughout the left lateral ventricle to safely reach the third ventricle. B: Photograph showing surface registration by laser scanning of the face. C: The endoscope is mounted on the robotic arm and can be easily handled by the surgeon

(Fig. 5). The mean surgical duration of DBS procedures was 415 minutes. There were no intraoperative complications. In 1 patient, the device was removed due to infection.

As already described for SEEG, we measured TPLE considering a $\mathrm{Y}$ value $=0$, as the depth of the electrode was conditioned by the intraoperative neurophysiological investigation. In our cases, the median TPLE was 1.67 $\mathrm{mm}$ (mean $1.59 \pm 0.52 \mathrm{~mm}$ ) for the left leads and $1.57 \mathrm{~mm}$ (mean $1.57 \pm 0.14 \mathrm{~mm}$ ) for the right electrodes.

Twelve patients affected by primary or secondary dystonia underwent bilateral pallidotomy. The mean surgical time was 287.7 minutes. In these cases, the left median TPLE was $1.27 \mathrm{~mm}$ (mean $1.41 \pm 0.28 \mathrm{~mm}$ ), and the median right TPLE was $1.34 \mathrm{~mm}$ (mean $1.48 \pm 0.38 \mathrm{~mm}$ ). No postoperative complications occurred.

\section{Discussion}

To the best of our knowledge, this is the largest reported series of pediatric neurosurgical cases that were performed using a robotic support. We used the ROSA system to assist 128 surgical procedures performed in 116 children, including implantation of SEEG electrodes, neuroendoscopy, tumor biopsy, functional procedures (DBS and pallidotomy), and other stereotactic approaches. In all cases, the robot allowed us to reach the planned target and to provide stable support to the instruments during the operative phase. The overall surgical success rate was $97.7 \%$, with a $3.9 \%$ rate of early clinical transient complications.

\section{General Considerations}

The ROSA system is an image-guided device with advanced navigation functions and haptic capabilities for both stereotactic and nonstereotactic approaches. ${ }^{12}$ According to the classification of Nathoo et al., ROSA belongs to both categories of supervisory-controlled and
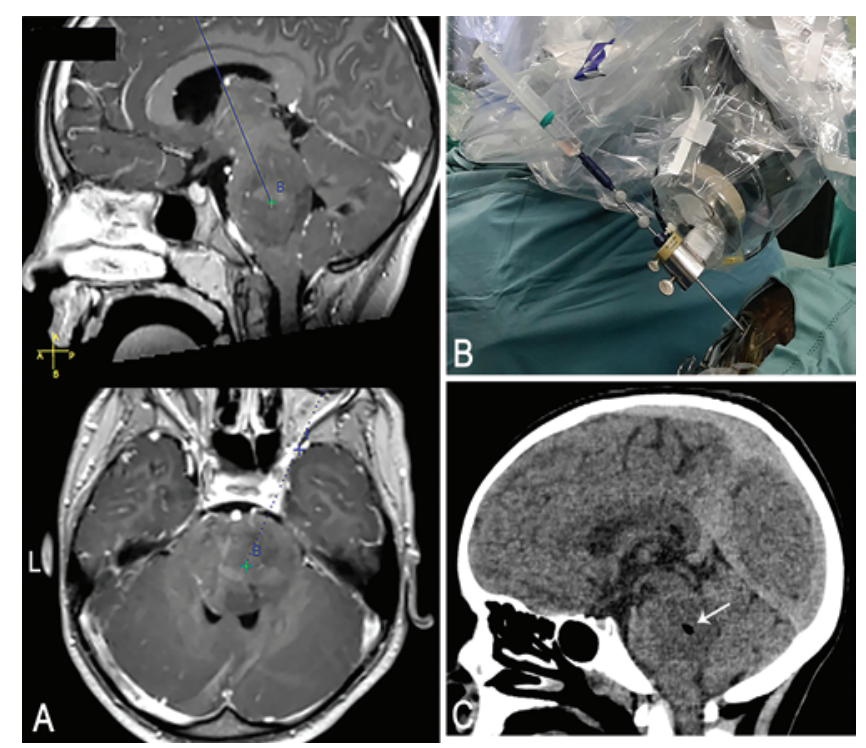

FIG. 4. A: Preoperative planning of a left transfrontal stereotactic biopsy of a diffuse intrapontine glioma in a 6-year-old girl. B: Intraoperative view of the biopsy needle, mounted on the robotic arm. C: Postoperative CT scan demonstrating the correct targeting of the biopsy (arrow).

shared-controlled systems.$^{48}$ In this modality, the surgeon, after offline planning, can either supervise the robot performing autonomously the motion or directly control and move the surgical instrument during the procedure.

During the planning phase, the safest trajectory can be calculated on MR images with respect to the vessels and eloquent deep structures. In this way, the surgical team can discuss the best surgical strategy before surgery, with a positive impact on the surgical and anesthesiological planning. The acquired plan is then transferred to analogous software included in the robotic system, and registration is performed in the operating room.

The device can perform all types of registrations: frame-based registration, frameless fiducial marker (skin or bone) registration, and frameless surface registration, depending on the location of the disease and the selected surgical approach. ${ }^{38,39}$ We have mainly used frameless surface registration, and bone fiducial registration only for DBS procedures, as indicated by the manufacturer. Moreover, as suggested in the literature, ${ }^{5}$ we did not observe any influence of other factors (such as the visual system, the height of the camera from the surface, and illumination) on the robot's accuracy.

Because of its haptic properties, the robot can be used not only for stereotactic procedures, but also to support and guide several surgical instruments during nonstereotactic approaches through $6 \mathrm{df}$ and in 3 possible interaction modes (axial, isocentric, and free). As demonstrated, the degrees of freedom value positively correlates with the time and error rate of the procedure. ${ }^{12}$

In the following sections, the main applications of the ROSA system are briefly discussed.

\section{Electrode Implantation for SEEG Recording}

SEEG recording through intracerebral electrodes is presently considered to be the gold-standard technique for 

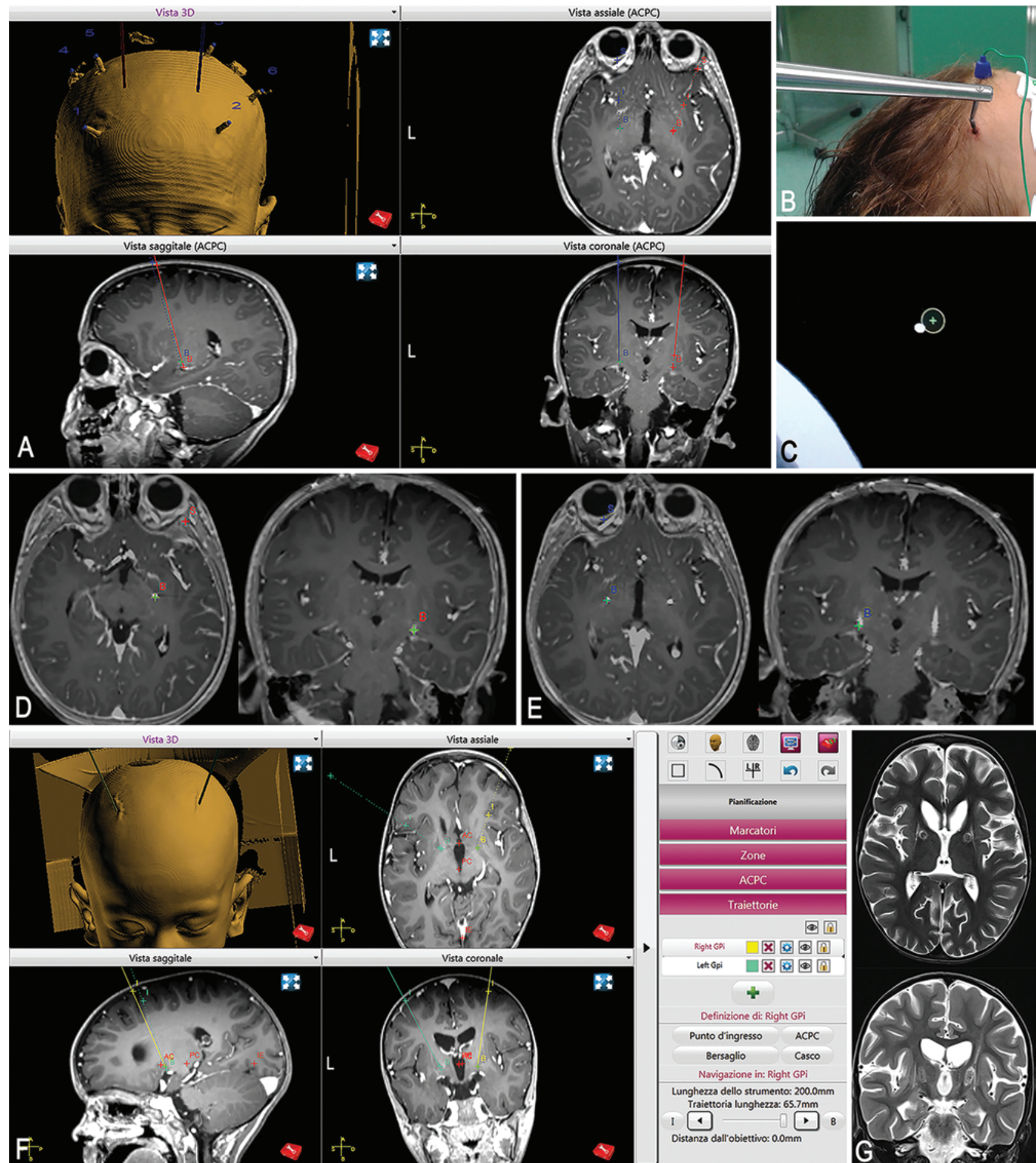

R

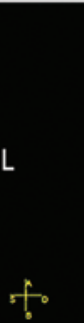

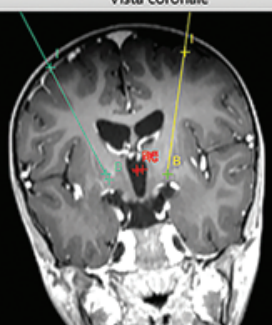

F
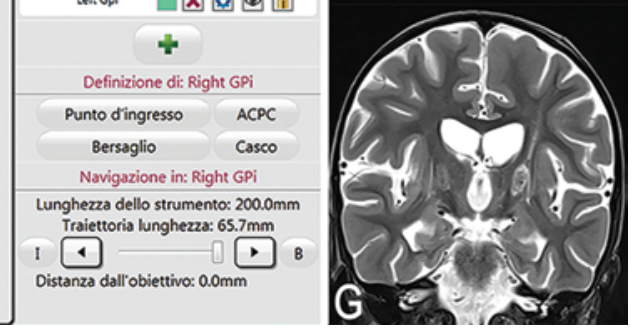

FIG. 5. A-C: ROSA-guided DBS in a 6-year-old girl affected by drug-resistant generalized dystonic-dyskinetic syndrome. Preoperative planning of the bilateral trajectory to the GPI, according to the anterior commissure-posterior commissure coordinates. The day before the procedure, the patient underwent implantation of 5 bone fiducial markers, as shown in the 3D view (A). During the registration phase, a specific pointer touches the fiducial tip (B). The accuracy of the registration is verified on a CT scan (C). $\mathrm{D}$ and $\mathrm{E}$ : Registration of the preoperative MR image with the postoperative CT scan, demonstrating the correct positioning of DBS electrodes on the right (D) and left (E) sides, according to the planned trajectory. F and G: Planning of bilateral pallidotomy in a 4-year-old boy with secondary dystonia (F). Postoperative CT scan demonstrating the correct site of the thermocoagulation at the level of the GPI, without complications $(\mathrm{G})$. 
preoperative workup in patients with drug-resistant epilepsy, in whom the epileptogenic zone cannot be detected using the current noninvasive methods. In fact, compared with other invasive approaches, such as subdural grids, SEEG provides a more accurate direct intracerebral recording of the electrical activity coming from superficial and deep structures, leading to a precise identification and delimitation of the epileptogenic zone. ${ }^{14,15,16,20}$ Moreover, depth electrodes are used not only to record electrophysiological ictal and interictal activity, but also to functionally map the brain area where they are inserted, which is crucial when epileptogenic foci are supposed to be adjacent to eloquent brain territories.

Over the last decade, due to the development of neuronavigation systems and robotic technologies, the traditional Talairach stereotactic frame-based SEEG methodology has been implemented, in terms of accuracy and safety, with a more multimodal approach and with frameless implantation procedures. ${ }^{14,17}$

So far, the application of ROSA in supporting SEEG has been described in some preliminary cases in adult and pediatric patients. Hughes et al. presented promising results from robot-assisted procedures performed in 4 adults (GL Hughes et al: Frameless stereotactic robot assistance in epilepsy surgery: preliminary results. Presentation to the Congress of Neurological Surgeons, Washington DC, October 1-6, 2011). In 2010, Ferrand-Sorbets et al. described good results in 15 children who underwent placement of 172 electrodes under ROSA control, also for bilateral exploration (S Ferrand Sorbets et al: Frameless stereotactic robot-guided placement of depth electrodes for stereo-electroencephalography in the presurgical evaluation of children with drug-resistant focal epilepsy. Presentation to the Congress of Neurological Surgeons, San Francisco, CA, 2010). Later, the same group reported the efficacy of SEEG in presurgical evaluation in an extended series of 65 children with drug-resistant epilepsy. Even if the surgical method was not specifically discussed in this paper, the results indicated a positive contribution of the robotic technique in optimizing diagnostic accuracy. ${ }^{63}$

We implanted a total of 386 electrodes in 36 children (mean 11 electrodes per patient, range 6-16) (Fig. 1). Four patients underwent a second SEEG procedure, because the first exploration was not conclusive about the definition of the epileptogenic network. The median errors were 1.5 $\mathrm{mm}$ at the entry point, and $1.96 \mathrm{~mm}$ at the target point. Recently, González-Martínez et al. reported a median EPLE of $1.2 \mathrm{~mm}$ and TPLE of $1.7 \mathrm{~mm}$ (major bleeding: 1\%) for 500 electrodes implanted at the Cleveland Clinic. ${ }^{29}$ Moreover, the patient series by Cardinale accounted for 1050 electrodes implanted under assistance of a different robotic system and frame-based registration (EPLE: $0.78 \mathrm{~mm}$, complications: $0 \%) \cdot{ }^{14}$ Although our results are slightly different in comparison with these groups, in our series we did not note any hemorrhagic intracranial complications, and we noted a reduction in the median error value with time, which is likely due to a progressive learning curve. In fact, in the last 15 cases, we observed an EPLE and a TPLE of $1.12 \mathrm{~mm}$ and $1.50 \mathrm{~mm}$, respectively.

As previously described, in addition to the learning curve, different factors can influence the discrepancy be- tween the planned trajectory and the actual one, including the inaccuracy of the CT scan merging process and the frameless optical tracking system, the possible skull shift during the bone drilling, especially when the planned trajectory is tangential to the skull surface, and the nonuse of the stylet to insert the electrodes. ${ }^{54}$

\section{Endoscopy-Guided Procedures}

Neuroendoscopy is a common treatment, with several possible applications in both adult and pediatric patients, such as management of obstructive hydrocephalus and intracranial cysts, resection or biopsy of tumors, and malformations. ${ }^{19,27,69}$

The use of robotic assistance for endoscopic approaches was first described by Benabid et al. in 1992. ${ }^{8}$ In 2002, Zimmermann reported encouraging preliminary data for 3 patients who underwent neuroendoscopic procedures under robotic assistance. ${ }^{73}$ These positive results were confirmed in a subsequent series of 6 adults affected by triventricular obstructive hydrocephalus, who underwent robot-assisted endoscopic third ventriculostomy. ${ }^{34,72} \mathrm{Pe}-$ diatric patients often represent a particular challenge, not only because of the high incidence of hydrocephalus-related diseases, but also for the frequent finding of an altered ventricular anatomy due to morphological variants or pathological distortions.

We performed 42 endoscopic procedures under ROSA guidance to manage different diseases, including secondary obstructive hydrocephalus (7 cases), arachnoid cyst (3 cases), intraventricular tumors (8 cases), and HHs (24 cases).

Despite the well-documented application of nonrobotic stereotactic approaches in neuroendoscopy, ${ }^{46,53,55}$ we opted for the robotic assistance to validate this technique in easier cases, (e.g., third ventriculostomy for obstructive hydrocephalus), especially at the beginning of our experience. More recently, we progressively changed the indications to more complex cases. In fact, pediatric patients often constitute a special challenge, not only because of the high incidence of hydrocephalus-related diseases, but also for the frequent finding of a tight ventricular system, deeply located lesions, and altered ventricular anatomy due to a morphological variant or pathological distortion.

In all of these cases, the robotic system guided the endoscope to the planned target. ${ }^{72,73}$ After reaching the ventricular or intracystic space, we switched the control of the robotic arm to the isocentric or manual mode for more precise and stable control of the instrument, according to freer trajectories, including rotations and axial translations.$^{69}$ For example, for 2 patients in whom an excluded ventricle developed due to previous shunt malfunction, shunt infection, or multiloculated hydrocephalus, despite the limited ventricular sizes, we successfully performed a ROSA-guided endoscopic laser septostomy to restore the correct CSF circulation (Fig. 2).

Nine patients underwent transventricular biopsy for tumor located in or pushing into the ventricle. Histological diagnosis was achieved in all patients, without complications. The positive diagnostic yield of neuroendoscopy for brain tumors has been previously documented ${ }^{58}$ Moreover, similar to nonrobotic stereotactic methods, the ROSA de- 
vice offers the possibility of verifying, during the entire procedure, the position of the endoscope through MRI, in addition to the direct endoscopic view.

HHs are congenital benign lesions, often associated with a typical medically resistant epileptic syndrome. Endoscopic disconnection represents an effective treatment for HHs, especially when the lesion bulges into the third ventricle. ${ }^{18,22,24}$ Moreover, in cases of persistent seizures, repetition of disconnection can be safely proposed to improve the epilepsy outcome. ${ }^{22,24,50}$ In this context, promising results of laser disconnection under ROSA assistance have been recently reported, ${ }^{13,50}$ and the possible use of the ROSA system in frameless stereotactic laser ablation as well has been described in an adult patient in whom stereotactic radiosurgery for $\mathrm{HH}$ failed..$^{11}$

We used the ROSA system to treat $24 \mathrm{HH}$ cases (Fig. 3). In particular, the robot was useful in safely accessing the lateral ventricle, which is usually small in these cases, and in easily guiding the laser or the monopolar system when performing multiple coagulations, not only along the base of intraventricular $\mathrm{HH}$ bulging, but also within the lesion. We repeated the procedure twice in 2 patients and 3 times in 1 patient, without additional complications. We believe that a robot-assisted approach and a combined technique of both disconnection and intralesional coagulation may be safely proposed as the first and repeatable option for optimizing the quality of surgical treatment of $\mathrm{HH}$ and the outcome of epilepsy, while minimizing the risk of postoperative morbidity.

\section{Stereotactic Biopsy}

Stereotactic biopsy is a minimally invasive technique and is used with the aim of obtaining a reliable histological diagnosis, especially in cases of small and deeply located brain tumors, and avoiding adjunctive and permanent morbidity. ${ }^{26,31,47}$ Traditionally, 2 possible approaches, including frame-based and frameless techniques, have been used, and many authors have discussed the differences and relative advantages of the 2 techniques. In general, frameless biopsy offers more ergonomic properties and an easier surgical workflow, ${ }^{21,25,71}$ especially in cases of supratentorial tumors and lesions larger than $15 \mathrm{~mm}$., ${ }^{3,49,57}$ On the other hand, frame-based biopsy envisages a more complex surgical workflow,,$^{23,51,57}$ and it is still preferred in many centers for the deepest and smallest lesions, or for lesions located close to highly vascular areas, such as the pineal region. ${ }^{39}$

So far, robot-assisted biopsies have been routinely performed at only a few centers in a limited number of patients. $6,23,32,39$ Only one group has recently reported the use of the ROSA device for stereotactic biopsies in a series of 100 tumors with various locations and histological types. Different registration modalities have been adopted (frameless robotic surface registration, robotic bone fiducial marker registration, and scalp fiducial marker registration) for both supine and prone approaches. The authors reported a high diagnostic yield (97\%), with a small rate of postoperative bleeding (10\%, $2 \%$ symptomatic) and a general transient neurological morbidity $(6 \%))^{38,39}$

We performed 26 biopsies with ROSA assistance for deeply located lesions (Fig. 4). We used surface registra- tion; all patients were in the supine position. A histological diagnosis was achieved in $96 \%$ of procedures. In 4 cases of cystic tumor component causing mass effect, we aspirated the cystic content through the biopsy needle. Similar to other procedures, we recorded a progressive reduction in surgery duration (mean time 122.7 minutes), mainly because of the vast experience gained with ROSA assistance and a faster registration phase. There were no deaths, infections, or permanent postsurgical sequelae. These results are in line with those of Lefranc et al..$^{38,39}$ and other groups that have reported a complication rate ranging between $0 \%$ and $9.3 \% .9,28,36,57$

In summary, our results confirm that stereotactic biopsy under ROSA guidance combines the advantages of both frameless and frame-based methods, in terms of technical aspects, timing, and diagnostic accuracy.

\section{Functional Procedures}

DBS and pallidotomy are common treatments, with several applications for movement disorders and psychiatric diseases. ${ }^{43,67,74}$ In contrast to SEEG, in which the goal is electrical exploration of superficial and deep brain regions along the implanted electrode according to the epileptogenic hypothesis, in DBS and pallidotomy the focus is the correct target identification. For this reason, these procedures require the utmost accuracy of lead placement in order to achieve the best clinical efficacy while avoiding side effects. ${ }^{10,44,60,64}$

In our preliminary experience, we treated 3 patients for primary dystonia ( 2 cases) and behavioral disorder (1 case). In all cases, we used bone fiducial registration. We registered a mean EPLE of $1.59 \pm 0.52 \mathrm{~mm}$ and TPLE of $1.57 \pm 0.14 \mathrm{~mm}$ (Fig. 5A-E).

The application of robotic technology in DBS procedures has already been reported with the use of the NeuroMate robot (Renishaw Inc.). ${ }^{66}$ Recently, von Langsdorff et al. evaluated the accuracy of the system in vitro and in vivo and registered mean values of $0.44 \pm 0.23 \mathrm{~mm}$ for in vitro application and of $0.86 \pm 0.32 \mathrm{~mm}$ during in vivo procedures. ${ }^{68}$

Moreover, our results are acceptable, in comparison with traditional frame-based and frameless techniques for deep electrode positioning. In fact, a previous comparative evaluation of the in vitro targeting accuracy of 4 framebased systems showed a mean error ranging between 1.7 and $1.9 \mathrm{~mm},{ }^{41}$ and a mean targeting error of $1.4 \pm 0.9 \mathrm{~mm}$ was found in a large clinical series of frame-based DBS procedures. ${ }^{33}$ In a later review regarding frameless stereotactic methods, Widmann et al. reported a mean target positioning error ranging between 1.1 and $1.3 \mathrm{~mm}$ for phantom studies, and between 1.99 and $3.2 \mathrm{~mm}$ for clinical observations, depending on the adopted targeting device. ${ }^{70}$ More recently, Bot et al. compared the accuracy between the Nexframe and the Leksell frame after location of 194 DBS leads, accounting for mean Euclidean errors of 2.71 \pm 1.23 and $2.63 \pm 1.07 \mathrm{~mm}$, respectively. ${ }^{10}$

In one of our cases, we removed the implant due to a device-related infection. As previously reported, ${ }^{52,59}$ the incidence of DBS complications may be even greater in pediatric patients, mainly due to soft-tissue coverage and poor general condition, such as in cases of status dystonicus. ${ }^{43}$ 
For this reason, pallidotomy represents a viable alternative for the management of movement disorders, especially in children in severe clinical condition, with the advantage of a decreased need of specialists and clinical follow-up and a lower risk of infection.

In our series, 11 dystonic patients underwent ROSAassisted pallidotomy (Fig. 5F and G). The mean EPLE and TPLE were $1.41 \pm 0.28 \mathrm{~mm}$ and $1.48 \pm 0.38 \mathrm{~mm}$, respectively, without complications. Refractory status dystonicus developed in 5 of these patients. Our group previously described the efficacy of pallidotomy in restoring the prestatus dystonicus condition, by controlling dystonic postures and movements of trunk and limbs. ${ }^{43}$

\section{Conclusions}

In this study, we demonstrated the usefulness of robot technology in supporting the neurosurgical management of several pediatric diseases. The ROSA system combines human decision making with the accuracy of machine technology by improving ergonomics, visualization, and the haptic ability of the surgeon, especially in cases of smaller targets, narrower corridors, and procedures requiring the highest level of precision, such as functional procedures and electrode implantation. Our results showed the versatility of the ROSA device, which, given the possibility to integrate different tools, improves the safety and feasibility of several minimally invasive procedures, while minimizing risks and surgical time. Further studies, involving larger case series, are needed to validate previous results, to improve current technology, and to optimize the impact of robotic stereotactic systems on the quality of neurosurgical procedures.

\section{Acknowledgments}

We are grateful to Ms. Paola Volpi for her valuable support in the language revision of the manuscript.

\section{References}

1. Aaronson OS, Tulipan NB, Cywes R, Sundell HW, Davis GH, Bruner JP, et al: Robot-assisted endoscopic intrauterine myelomeningocele repair: a feasibility study. Pediatr Neurosurg 36:85-89, 2002

2. Adler JR Jr, Murphy MJ, Chang SD, Hancock SL: Imageguided robotic radiosurgery. Neurosurgery 44:1299-1307, 1999

3. Air EL, Leach JL, Warnick RE, McPherson CM: Comparing the risks of frameless stereotactic biopsy in eloquent and noneloquent regions of the brain: a retrospective review of 284 cases. J Neurosurg 111:820-824, 2009

4. Arata J, Tada Y, Kozuka H, Wada T, Saito Y, Ikedo N, et al: Neurosurgical robotic system for brain tumor removal. Int J CARS 6:375-385, 2011

5. Awang MS, Abdullah MZ: Robotic neurosurgery: a preliminary study using an active vision-guided robotic arm for bone drilling and endoscopic manoeuvres. Malays J Med Sci 18:53-57, 2011

6. Bekelis K, Radwan TA, Desai A, Roberts DW: Frameless robotically targeted stereotactic brain biopsy: feasibility, diagnostic yield, and safety. J Neurosurg 116:1002-1006, 2012

7. Benabid AL, Cinquin P, Lavalle S, Le Bas JF, Demongeot J, de Rougemont J: Computer-driven robot for stereotactic sur- gery connected to CT scan and magnetic resonance imaging. Technological design and preliminary results. Appl Neurophysiol 50:153-154, 1987

8. Benabid AL, Lavallee S, Hoffmann D, Cinquin P, Demongeot J, Danel F: Potential use of robots in endoscopic neurosurgery. Acta Neurochir Suppl (Wien) 54:93-97, 1992

9. Bernstein M, Parrent AG: Complications of CT-guided stereotactic biopsy of intra-axial brain lesions. J Neurosurg 81:165-168, 1994

10. Bot M, van den Munckhof P, Bakay R, Sierens D, Stebbins G, Verhagen Metman L: Analysis of stereotactic accuracy in patients undergoing deep brain stimulation using Nexframe and the Leksell frame. Stereotact Funct Neurosurg 93:316-325, 2015

11. Brandmeir N, Acharya V, Sather M: Robot assisted stereotactic laser ablation for a radiosurgery resistant hypothalamic hamartoma. Cureus 8:e581, 2016

12. Brodie J, Eljamel S: Evaluation of a neurosurgical robotic system to make accurate burr holes. Int J Med Robot 7:101106, 2011

13. Calisto A, Dorfmüller G, Fohlen M, Bulteau C, Conti A, Delalande O: Endoscopic disconnection of hypothalamic hamartomas: safety and feasibility of robot-assisted, thulium laser-based procedures. J Neurosurg Pediatr 14:563-572, 2014

14. Cardinale F: Stereoelectroencephalography: application accuracy, efficacy, and safety. World Neurosurg 94:570-571, 2016

15. Cardinale F: Stereoelectroencephalography for insular-opercular/perisylvian epilepsy. J Neurosurg Pediatr 19:271-272, 2017 (Letter)

16. Cardinale F, Casaceli G, Raneri F, Miller J, Lo Russo G: Implantation of stereoelectroencephalography electrodes: a systematic review. J Clin Neurophysiol 33:490-502, 2016

17. Cardinale F, Cossu M, Castana L, Casaceli G, Schiariti MP, Miserocchi A, et al: Stereoelectroencephalography: surgical methodology, safety, and stereotactic application accuracy in 500 procedures. Neurosurgery 72:353-366, 2013

18. Choi JU, Yang KH, Kim TG, Chang JH, Chang JW, Lee BI, et al: Endoscopic disconnection for hypothalamic hamartoma with intractable seizure. Report of four cases. J Neurosurg 100 (5 Suppl Pediatrics):506-511, 2004

19. Constantini S, Sgouros S, Kulkarni A: Neuroendoscopy in the youngest age group. World Neurosurg 79 (2 Suppl):S23. e1-S23.e11, 2013

20. Cossu M, Schiariti M, Francione S, Fuschillo D, Gozzo F, Nobili L, et al: Stereoelectroencephalography in the presurgical evaluation of focal epilepsy in infancy and early childhood. J Neurosurg Pediatr 9:290-300, 2012

21. Dammers R, Haitsma IK, Schouten JW, Kros JM, Avezaat CJJ, Vincent AJPE: Safety and efficacy of frameless and frame-based intracranial biopsy techniques. Acta Neurochir (Wien) 150:23-29, 2008

22. Delalande O, Fohlen M: Disconnecting surgical treatment of hypothalamic hamartoma in children and adults with refractory epilepsy and proposal of a new classification. Neurol Med Chir (Tokyo) 43:61-68, 2003

23. Dellaretti M, Reyns N, Touzet G, Dubois F, Gusmão S, Pereira JLB, et al: Stereotactic biopsy for brainstem tumors: comparison of transcerebellar with transfrontal approach. Stereotact Funct Neurosurg 90:79-83, 2012

24. Dorfmüller G, Fohlen M, Bulteau C, Delalande O: [Surgical disconnection of hypothalamic hamartomas.] Neurochirurgie 54:315-319, $2008(\mathrm{Fr})$

25. Dorward NL, Alberti O, Palmer JD, Kitchen ND, Thomas DG: Accuracy of true frameless stereotaxy: in vivo measurement and laboratory phantom studies. Technical note. J Neurosurg 90:160-168, 1999

26. Dorward NL, Paleologos TS, Alberti O, Thomas DG: The 
advantages of frameless stereotactic biopsy over frame-based biopsy. Br J Neurosurg 16:110-118, 2002

27. Durnford AJ, Kirkham FJ, Mathad N, Sparrow OC: Endoscopic third ventriculostomy in the treatment of childhood hydrocephalus: validation of a success score that predicts long-term outcome. J Neurosurg Pediatr 8:489493, 2011

28. Field M, Witham TF, Flickinger JC, Kondziolka D, Lunsford LD: Comprehensive assessment of hemorrhage risks and outcomes after stereotactic brain biopsy. J Neurosurg 94:545-551, 2001

29. González-Martínez J, Bulacio J, Thompson S, Gale J, Smithason S, Najm I, et al: Technique, results, and complications related to robot-assisted stereoelectroencephalography. Neurosurgery 78:169-180, 2016

30. Gonzalez-Martinez J, Vadera S, Mullin J, Enatsu R, Alexopoulos AV, Patwardhan R, et al: Robot-assisted stereotactic laser ablation in medically intractable epilepsy: operative technique. Neurosurgery 10 (Suppl 2):167-173, 2014

31. Grossman R, Sadetzki S, Spiegelmann R, Ram Z: Haemorrhagic complications and the incidence of asymptomatic bleeding associated with stereotactic brain biopsies. Acta Neurochir (Wien) 147:627-631, 2005

32. Haegelen C, Touzet G, Reyns N, Maurage CA, Ayachi M, Blond S: Stereotactic robot-guided biopsies of brain stem lesions: experience with 15 cases. Neurochirurgie 56:363367, 2010

33. Holl EM, Petersen EA, Foltynie T, Martinez-Torres I, Limousin P, Hariz MI, et al: Improving targeting in image-guided frame-based deep brain stimulation. Neurosurgery 67 (2 Suppl Operative):437-447, 2010

34. Hoshide R, Calayag M, Meltzer HS, Levy ML, Gonda DD: Robot-assisted endoscopic third ventriculostomy. Neurosurgery 63 (Suppl 1):204, 2016

35. Kelly PJ, Alker GJ Jr, Goerss S: Computer-assisted stereotactic microsurgery for the treatment of intracranial neoplasms. Neurosurgery 10:324-331, 1982

36. Krieger MD, Chandrasoma PT, Zee CS, Apuzzo ML: Role of stereotactic biopsy in the diagnosis and management of brain tumors. Semin Surg Oncol 14:13-25, 1998

37. Kwoh YS, Hou J, Jonckheere EA, Hayati S: A robot with improved absolute positioning accuracy for CT guided stereotactic brain surgery. IEEE Trans Biomed Eng 35:153-160, 1988

38. Lefranc M, Capel C, Pruvot AS, Fichten A, Desenclos C, Toussaint $\mathrm{P}$, et al: The impact of the reference imaging modality, registration method and intraoperative flat-panel computed tomography on the accuracy of the ROSA ${ }^{\circledR}$ stereotactic robot. Stereotact Funct Neurosurg 92:242-250, 2014

39. Lefranc M, Touzet G, Caron S, Maurage CA, Assaker R, Blond S: Are stereotactic sample biopsies still of value in the modern management of pineal region tumours? Lessons from a single-department, retrospective series. Acta Neurochir (Wien) 153:1111-1122, 2011

40. Louw DF, Fielding T, McBeth PB, Gregoris D, Newhook P, Sutherland GR: Surgical robotics: a review and neurosurgical prototype development. Neurosurgery 54:525-537, 2004

41. Maciunas RJ, Galloway RL Jr, Latimer JW: The application accuracy of stereotactic frames. Neurosurgery 35:682-695, 1994

42. Maddhai Y, Zareinia K, Gan LS, Sutherland C, Lama S, Sutherland GR: Treatment of glioma using neuroArm Surgical System. BioMed Res Int 9734512:2016, 2016

43. Marras CE, Rizzi M, Cantonetti L, Rebessi E, De Benedictis A, Portaluri F, et al: Pallidotomy for medically refractory status dystonicus in childhood. Dev Med Child Neurol 56:649-656, 2014

44. Mazzone P, Arena P, Cantelli L, Spampinato G, Sposato S,
Cozzolino S, et al: Experimental new automatic tools for robotic stereotactic neurosurgery: towards "no hands" procedure of leads implantation into a brain target. J Neural Transm (Vienna) 123:737-750, 2016

45. McBeth PB, Louw DF, Rizun PR, Sutherland GR: Robotics in neurosurgery. Am J Surg 188 (4A Suppl):68S-75S, 2004

46. McGirt MJ, Woodworth GF, Coon AL, Frazier JM, Amundson E, Garonzik I, et al: Independent predictors of morbidity after image-guided stereotactic brain biopsy: a risk assessment of 270 cases. J Neurosurg 102:897-901, 2005

47. McMillen JL, Vonau M, Wood MJ: Pinless frameless electromagnetic image-guided neuroendoscopy in children. Childs Nerv Syst 26:871-878, 2010

48. Nathoo N, Cavuşoğlu MC, Vogelbaum MA, Barnett GH: In touch with robotics: neurosurgery for the future. Neurosurgery 56:421-433, 2005

49. Owen CM, Linskey ME: Frame-based stereotaxy in a frameless era: current capabilities, relative role, and the positiveand negative predictive values of blood through the needle. $\mathbf{J}$ Neurooncol 93:139-149, 2009

50. Procaccini E, Dorfmüller G, Fohlen M, Bulteau C, Delalande $O$ : Surgical management of hypothalamic hamartomas with epilepsy: the stereoendoscopic approach. Neurosurgery 59 (4 Suppl 2):ONS336-ONS346, 2006

51. Roujeau T, Machado G, Garnett MR, Miquel C, Puget S, Geoerger B, et al: Stereotactic biopsy of diffuse pontine lesions in children. J Neurosurg 107 (1 Suppl):1-4, 2007

52. Rughani AI, Hodaie M, Lozano AM: Acute complications of movement disorders surgery: effects of age and comorbidities. Mov Disord 28:1661-1667, 2013

53. Sangra M, Clark S, Hayhurst C, Mallucci C: Electromagnetic-guided neuroendoscopy in the pediatric population. $\mathbf{J}$ Neurosurg Pediatr 3:325-330, 2009

54. Schicho K, Figl M, Seemann R, Ewers R, Lambrecht JT, Wagner A, et al: Accuracy of treatment planning based on stereolithography in computer assisted surgery. Med Phys 33:3408-3417, 2006

55. Schroeder HWS, Wagner W, Tschiltschke W, Gaab MR: Frameless neuronavigation in intracranial endoscopic neurosurgery. J Neurosurg 94:72-79, 2001

56. Shah J, Mackay S, Rockall T, Vale J, Darzi A: 'Urobotics': robots in urology. BJU Int 88:313-320, 2001

57. Smith JS, Quiñones-Hinojosa A, Barbaro NM, McDermott MW: Frame-based stereotactic biopsy remains an important diagnostic tool with distinct advantages over frameless stereotactic biopsy. J Neurooncol 73:173-179, 2005

58. Somji M, Badhiwala J, McLellan A, Kulkarni AV: Diagnostic yield, morbidity, and mortality of intraventricular neuroendoscopic biopsy: systematic review and meta-analysis. World Neurosurg 85:315-324, 324.e1-324.e2, 2016

59. Soo TM, Bernstein M, Provias J, Tasker R, Lozano A, Guha A: Failed stereotactic biopsy in a series of 518 cases. Stereotact Funct Neurosurg 64:183-196, 1995

60. Starr PA, Martin AJ, Larson PS: Implantation of deep brain stimulator electrodes using interventional MRI. Neurosurg Clin N Am 20:193-203, 2009

61. Sutherland GR, Latour I, Greer AD, Fielding T, Feil G, Newhook P: An image-guided magnetic resonance-compatible surgical robot. Neurosurgery 62:286-293, 2008

62. Sutherland GR, Maddahi Y, Gan LS, Lama S, Zareinia K: Robotics in the neurosurgical treatment of glioma. Surg Neurol Int 6 (Suppl 1):S1-S8, 2015

63. Taussig D, Chipaux M, Lebas A, Fohlen M, Bulteau C, Ternier J, et al: Stereo-electroencephalography (SEEG) in 65 children: an effective and safe diagnostic method for pre-surgical diagnosis, independent of age. Epileptic Disord 16:280-295, 2014

64. Terao T, Takahashi H, Yokochi F, Taniguchi M, Okiyama R, Hamada I: Hemorrhagic complication of stereotactic surgery 
in patients with movement disorders. J Neurosurg 98:12411246, 2003

65. Tsai EC, Santoreneos S, Rutka JT: Tumors of the skull base in children: review of tumor types and management strategies. Neurosurg Focus 12(5):e1, 2002

66. Varma TR, Eldridge P: Use of the NeuroMate stereotactic robot in a frameless mode for functional neurosurgery. Int $\mathbf{J}$ Med Robot 2:107-113, 2006

67. Vergani F, Landi A, Pirillo D, Cilia R, Antonini A, Sganzerla EP: Surgical, medical, and hardware adverse events in a series of 141 patients undergoing subthalamic deep brain stimulation for Parkinson disease. World Neurosurg 73:338-344, 2010

68. von Langsdorff D, Paquis P, Fontaine D: In vivo measurement of the frame-based application accuracy of the Neuromate neurosurgical robot. J Neurosurg 122:191-194, 2015

69. Vougioukas VI, Hubbe U, Hochmuth A, Gellrich NC, van Velthoven V: Perspectives and limitations of image-guided neurosurgery in pediatric patients. Childs Nerv Syst 19:783791,2003

70. Widmann G, Schullian P, Ortler M, Bale R: Frameless stereotactic targeting devices: technical features, targeting errors and clinical results. Int J Med Robot 8:1-16, 2012

71. Woodworth GF, McGirt MJ, Samdani A, Garonzik I, Olivi A, Weingart JD: Frameless image-guided stereotactic brain biopsy procedure: diagnostic yield, surgical morbidity, and comparison with the frame-based technique. J Neurosurg 104:233-237, 2006

72. Zimmermann M, Krishnan R, Raabe A, Seifert V: Robot-assisted navigated endoscopic ventriculostomy: implementation of a new technology and first clinical results. Acta Neurochir (Wien) 146:697-704, 2004

73. Zimmermann M, Krishnan R, Raabe A, Seifert V: Robotassisted navigated neuroendoscopy. Neurosurgery 51:14461452,2002
74. Zorzi G, Marras C, Nardocci N, Franzini A, Chiapparini L, Maccagnano E, et al: Stimulation of the globus pallidus internus for childhood-onset dystonia. Mov Disord 20:11941200,2005

\section{Disclosures}

The authors report no conflict of interest concerning the materials or methods used in this study or the findings specified in this paper.

\section{Author Contributions}

Conception and design: Carai, De Benedictis, Trezza, Procaccini, Messina, Randi, Cossu, Esposito, Palma, Marras. Acquisition of data: Carai, De Benedictis, Trezza, Genovese, Procaccini, Messina, Randi, Cossu, Esposito, Palma, Marras. Analysis and interpretation of data: Carai, De Benedictis, Trezza, Messina, Randi, Cossu, Esposito, Palma, Rizzi, Marras. Drafting the article: Carai, De Benedictis, Trezza, Procaccini. Critically revising the article: Carai, De Benedictis, Trezza, Amante, Rizzi, Marras. Reviewed submitted version of manuscript: Carai, De Benedictis, Trezza, Rizzi, Marras. Approved the final version of the manuscript on behalf of all authors: Carai. Statistical analysis: De Benedictis. Administrative/technical/material support: De Benedictis, Trezza, Genovese, Procaccini. Study supervision: De Benedictis, Trezza, Marras.

\section{Correspondence}

Andrea Carai, Department of Neuroscience, Neurosurgical Unit, Bambino Gesù Children's Hospital, IRCCS, 4, Piazza Sant'Onofrio, Rome 00165, Italy. email: andrea.carai@opbg.net. 